\title{
FREE PRODUCTS OF TOPOLOGICAL GROUPS WITH A CLOSED SUBGROUP AMALGAMATED
}

\author{
PETER NICKOLAS
}

(Received 15 April 1984; revised 1 December 1984)

Communicated by J. H. Rubinstein

\begin{abstract}
It is shown that if $\left\{G_{n}: n=1,2, \ldots\right\}$ is a countable family of Hausdorff $\mathrm{k}_{\omega}$-topological groups with a common closed subgroup $\mathrm{A}$, then the topological amalgamated free product ${ }_{A}{ }_{A} G_{n}$ exists and is a Hausdorff $\mathrm{k}_{\omega}$-topological group with each $G_{n}$ as a closed subgroup. A consequence is the theorem of La Martin that epimorphisms in the category of $\mathrm{k}_{\omega}$-topological groups have dense image.
\end{abstract}

1980 Mathematics subject classification (Amer. Math. Soc.): 22 A 05; secondary 20 E 06, 54 D 30, 18 A 20 .

\section{Introduction}

Let $\left\{G_{n}: n=1,2, \ldots\right\}$ be a countable family of $\mathrm{k}_{\omega}$-topological groups, each having a fixed topological group $\mathrm{A}$ as a closed subgroup. We show that ${ }_{A} G_{n}$, the free topological product of $\left\{G_{n}\right\}$ with $A$ amalgamated, exists, is a (Hausdorff) $\mathrm{k}_{\omega}$-group, and contains $G_{n}$ as a closed subgroup for each $n$.

Katz and Morris $[2,3,4]$ have already shown that an amalgamated product $G$ ${ }^{*} H$ of $\mathrm{k}_{\omega}$-groups is $\mathrm{k}_{\omega}$ whenever $A$ is in a class of closed subgroups, including those which are normal and those which are the product of a compact subgroup and a central subgroup. Our theorem clearly contains these results, and moreover yields another proof of La Martin's theorem that epimorphisms in the category of $\mathrm{k}_{\omega}$-groups have dense range ([6]; see also [9] and [11]).

(c) 1986 Australian Mathematical Society $0263-6115 / 86 \$ A 2.00+0.00$ 
The proof of our theorem has similarities to Ordman's proof [10] that the free $\mathrm{k}$-group on a $\mathrm{t}_{2} \mathrm{k}$-space exists and is a $\mathrm{t}_{2} \mathrm{k}$-group, and more especially to the proof of Brown and Hardy [1] that the universal topological groupoid on a $\mathbf{k}_{\omega}$-groupoid exists and is $\mathbf{k}_{\omega}$.

\section{The theorem}

Recall that a Hausdorff space $X$ is a $k_{\omega}$-space if it has the weak topology with respect to some increasing sequence of compact subsets $X_{1} \subseteq X_{2} \subseteq \cdots$ with union $X$; then we say that $\cup X_{n}$ is a $k_{\omega}$-decomposition of $X$. A topological group is a $k_{\omega}$-group if as a topological space it is $\mathrm{k}_{\omega}$. The appendix of [1] contains a useful list of the properties of $\mathbf{k}_{\omega}$-spaces.

Let $\left\{G_{\lambda}: \lambda \in \Lambda\right\}$ be a family of topological groups. Then we say that $\left(A,\left\{i_{\lambda}\right\}\right)$ is a common subgroup of the $G_{\lambda}$ if $A$ is a topological group and, for each $\lambda \in \Lambda$, $i_{\lambda}$ is a topological isomorphism of $A$ onto a subgroup of $G_{\lambda}$. We denote $i_{\lambda}(A)$ by $A_{\lambda}$, and the isomorphism $i_{\mu} i_{\lambda}^{-1}: A_{\lambda} \rightarrow A_{\mu}$, where $\lambda, \mu \in \Lambda$, is denoted by $i_{\lambda, \mu}$. The common subgroup is closed if $A_{\lambda}$ is closed in $G_{\lambda}$ for each $\lambda$.

The above isomorphisms, of course, simply serve to identify the various copies of $A$ in the $G_{\lambda}$. In purely algebraic arguments involving the amalgamated product it is often convenient to suppress these maps, and to regard $A$ as a subgroup of each $G_{\lambda}$ (cf. Chapter III, 12 of [8]); this can be done with advantage in the lemma below. In topological arguments, on the other hand, it is desirable to use the maps explicitly.

Definition (cf. $[2,3,4])$. Let $\left(A,\left\{i_{\lambda}\right\}\right)$ be a common subgroup of the topological groups $G_{\lambda}, \lambda \in \Lambda$. A topological group $G={ }^{*}{ }_{A} G_{\lambda}$ is the free product of $\left\{G_{\lambda}\right\}$ with $A$ amalgamated if

(i) $G_{\lambda}$ is a topological subgroup of $G$ for each $\lambda$,

(ii) $\cup_{\lambda} G_{\lambda}$ generates $G$ algebraically, and

(iii) for any topological group $H$ and any collection of continuous homomorphisms $\phi_{\lambda}: G_{\lambda} \rightarrow H$ which agree on $A$ (that is $\phi_{\lambda} i_{\lambda}=\phi_{\mu} i_{\mu}$ for all $\lambda$ and $\mu$ ), there exists a continuous homomorphism $\Phi: G \rightarrow H$ which extends each $\phi_{\lambda}$.

THEOREM. If $\left(A,\left\{i_{n}\right\}\right)$ is a common closed subgroup of the $k_{\omega}$-groups $G_{n}$, $n \in \mathbf{N}$, then $*_{A} G_{n}$ exists and is a (Hausdorff) $k_{\omega}$-group, with each $G_{n}$ as a closed subgroup.

Note that $A$ is also necessarily a $\mathrm{k}_{\omega}$-group.

The proof of the theorem occupies almost the remainder of the paper. 
Let $U=\sqcup_{n} G_{n}$ and $W=\sqcup_{n} U^{n}=\cup_{n} W_{n}$, where $W_{n}=\sqcup_{i=1}^{n} U^{i}$ (here $\sqcup$ denotes disjoint union (or the coproduct in the category of topological spaces), and $U^{n}$ denotes the Cartesian product $U \times \cdots \times U$ of $n$ copies of $U$ ). Clearly $U$ and $W$ are $k_{\omega}$-spaces. Let $G$ be the abstract amalgamated free product ${ }_{A} G_{n}$ of the $G_{n}$ with the $A_{n}$ amalgamated, and give $G$ the quotient topology under the map $p: W \rightarrow G$ which sends $\left(g_{1}, \ldots, g_{n}\right)$ to the product of $g_{1}, \ldots, g_{n}$ in $G$. We shall show that $G$ has all the properties required by the definition. The key to doing this is to show first that $G$ has a (Hausdorff) $\mathrm{k}_{\omega}$-topology, and for this we need the definition and lemma below.

For convenience, first define $\Omega: U \rightarrow \mathbf{N}$ by setting $\Omega(g)$, for $g \in U$, equal to the (unique) $n \in \mathbf{N}$ for which $g \in G_{n}$.

Definition. An $n$-tuple $\left(g_{1}, \ldots, g_{n}\right) \in W$ is reduced if $g_{j} \in G_{\Omega\left(g_{j}\right)} \backslash A_{\Omega\left(g_{j}\right)}$, $j=1, \ldots, n$, and if $\Omega\left(g_{j}\right) \neq \Omega\left(g_{j+1}\right), j=1, \ldots, n-1$.

Lemma. Let $\left(g_{1}, \ldots, g_{n}\right)$ and $\left(h_{1}, \ldots, h_{m}\right)$ be reduced elements of $W$. Then, writing $\omega(j)=\omega\left(g_{j}\right)$ for $j=1, \ldots, n$, we have $p\left(g_{1}, \ldots, g_{n}\right)=p\left(h_{1}, \ldots, h_{m}\right)$ if and only if

(i) $n=m$,

(ii) $\Omega\left(h_{j}\right)=\omega(j), j=1, \ldots, n$, and

(iii) $h_{1}^{-1} g_{1} \in A_{\omega(1)}$,

$h_{2}^{-1} i_{\omega(1), \omega(2)}\left(h_{1}^{-1} g_{1}\right) g_{2} \in A_{\omega(2)}$,

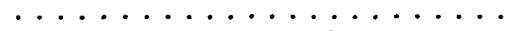

$h_{n-1}^{-1} i_{\omega(n-2), \omega(n-1)}\left(h_{n-2}^{-1} \cdots g_{n-2}\right) g_{n-1} \in A_{\omega(n-1)}$, and

$h_{n}^{-1} i_{\omega(n-1), \omega(n)}\left(h_{n-1}^{-1} \cdots g_{n-1}\right) g_{n}=1$.

Moreover, (i), (ii) and (iii) together imply that $p\left(g_{1}, \ldots, g_{n}\right)=p\left(h_{1}, \ldots, h_{m}\right)$, whether or not $\left(g_{1}, \ldots, g_{n}\right)$ and $\left(h_{1}, \ldots, h_{m}\right)$ are reduced.

Proof. Suppose $p\left(g_{1}, \ldots, g_{n}\right)=p\left(h_{1}, \ldots, h_{m}\right)$. By Chapter I of [8], we see that $p\left(g_{1}, \ldots, g_{n}\right)$ and $p\left(h_{1}, \ldots, h_{m}\right)$ have lengths $n$ and $m$, respectively, in $G$, so that $n=m$, proving (i).

Let $S_{k}(k \in \mathbf{N})$ be a complete set of left coset representatives for $A_{k}$ in $G_{k}$, with the representative of $A_{k}$ always taken to be 1 . Recall that for all $k, l \in \mathbf{N}$, $i_{k}(a)$ and $i_{l}(a)$ are identified as elements of $G$, for each $a \in A$. In the group $G_{\omega(1)}$, set

$$
g_{1}=s_{1} a_{1} \quad\left(s_{1} \in S_{\omega(1)} \backslash\{1\}, a_{1} \in A_{\omega(1)}\right),
$$

and in the group $G_{\omega(j)}, j=2, \ldots, n$, set

$$
i_{\omega(j-1), \omega(j)}\left(a_{j-1}\right) g_{j}=s_{j} a_{j} \quad\left(s_{j} \in S_{\omega(j)} \backslash\{1\}, a_{j} \in A_{\omega(j)}\right) .
$$


Then from the well-known algebraic structure of $G$ [8], we see that, in the group $G, g_{1} g_{2} \cdots g_{n}=s_{1} s_{2} \cdots s_{n} a_{n}$, and that the latter is the (uniquely-defined) normal form of $g_{1} g_{2} \cdots g_{n}$. Computing the normal form of $h_{1} h_{2} \cdots h_{n}$ similarly, we see that (writing $\left.\Omega^{\prime}(j)=\Omega\left(h_{j}\right), j=1, \ldots, n\right)$ we have

$$
h_{1}=s_{1}^{\prime} a_{1}^{\prime} \quad\left(s_{1}^{\prime} \in S_{\omega^{\prime}(1)} \backslash\{1\}, a_{1} \in A_{\omega^{\prime}(1)}\right)
$$

and, for $j=2, \ldots, n$,

$$
i_{\omega^{\prime}(j-1), \omega^{\prime}(j)}\left(a_{j-1}^{\prime}\right) h_{j}=s_{j}^{\prime} a_{j}^{\prime} \quad\left(s_{j}^{\prime} \in S_{\omega^{\prime}(j)} \backslash\{1\}, a_{j}^{\prime} \in A_{\omega^{\prime}(j)}\right),
$$

so that $h_{1} h_{2} \cdots h_{n}$ has normal form $s_{1}^{\prime} s_{2}^{\prime} \cdots s_{n}^{\prime} a_{n}^{\prime}$. Since each element of $G$ has a unique normal form, we must have $s_{j}=s_{j}^{\prime}, j=1, \ldots, n$, and $a_{n}=a_{n}^{\prime}$, and so $\omega(j)=\omega^{\prime}(j)$ for each $j$, proving (ii).

Combining (1) and (3) then shows that $h_{1}^{-1} g_{1}=\left(a_{1}^{\prime}\right)^{-1} a_{1} \in A_{\omega(1)}$, and from repeated combination of (2) and (4) it follows that $h_{j}^{-1} i_{\omega(j-1), \omega(j)}\left(h_{j-1}^{-1} \cdots\right.$ $\left.g_{j-1}\right) g_{j}=\left(a_{j}^{\prime}\right)^{-1} a_{j} \in A_{\omega(j)}, j=2, \ldots, n$. Thus (noting that $\left(a_{n}^{\prime}\right)^{-1} a_{n}=1$ ) we see that (iii) is true.

The remainder of the proof of the lemma follows along similar lines, again using the normal form, and the details are left to the reader.

Proposition. The graph $\Gamma$ of the equivalence relation defined by $p$ (that is, the set $\left.\left\{\left(w, w^{\prime}\right) \in W \times W: p(w)=p\left(w^{\prime}\right)\right\}\right)$ is closed in $W \times W$.

Proof. Clearly $W \times W$ has the weak topology with respect to the sets $W_{n} \times W_{n}$, and it suffices to show that $\Gamma_{n}=\Gamma \cap\left(W_{n} \times W_{n}\right)$ is closed in $W_{n} \times W_{n}$ for each $n$. The proof is by induction on $n$. We point out that the proof will not make use of the fact that the $G_{n}$ are $\mathbf{k}_{\omega}$; Hausdorffness is the only topological condition required.

Now $W_{1} \times W_{1}=U \times U=\sqcup_{j, k} G_{j} \times G_{k}$, and it is clear that

$$
\Gamma \cap\left(G_{j} \times G_{k}\right)= \begin{cases}\left\{(g, g): g \in G_{j}\right\}, & j=k, \\ \left\{\left(i_{j}(a), i_{k}(a)\right): a \in A\right\}, & j \neq k,\end{cases}
$$

which is closed for all $j$ and $k$, as each $G_{i}$ is Hausdorff and $A_{i}$ is closed in each $G_{i}$. Hence $\Gamma_{1}$ is closed in $W_{1} \times W_{1}$.

Suppose that $\Gamma_{n-1}$ is closed in $W_{n-1} \times W_{n-1}$ for some $n \geqslant 2$. We proceed to show that $\Gamma_{n}$ is closed in $W_{n} \times W_{n}$. This will be done by decomposing $W_{n} \times W_{n}$ into a disjoint union of smaller subspaces, and by showing that the intersection of $\Gamma_{n}$ with each of these is closed. To this end, we introduce some definitions.

For $k_{1}, \ldots, k_{n} \in \mathbf{N}$ define $K\left(k_{1}, \ldots, k_{n}\right)$ to be the set of $\left(g_{1}, \ldots, g_{n}, h_{1}, \ldots, h_{n}\right) \in G_{k_{1}} \times \cdots \times G_{k_{n}} \times G_{k_{1}} \times \cdots \times G_{k_{n}}$ such that $g_{1}, \ldots, g_{n}, h_{1}, \ldots, h_{n}$ satisfy all the conditions listed in (iii) of the lemma. It is straightforward to check that $K\left(k_{1}, \ldots, k_{n}\right)$ is a closed subset of the above product. 
Also for $k_{1}, \ldots, k_{n} \in \mathbf{N}$, we define certain classes of functions from subsets of $G_{k_{1}} \times \cdots \times G_{k_{n}}$ into $W_{n-1}$ as follows. First, if for any $p(1 \leqslant p \leqslant n-1), k_{p}$ and

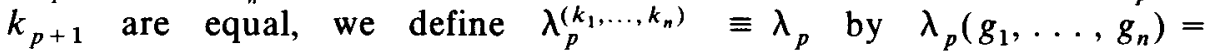
$\left(g_{1}, \ldots,\left(g_{p} g_{p+1}\right), \ldots, g_{n}\right) \in W_{n-1}$ for each $\left(g_{1}, \ldots, g_{n}\right) \in G_{k_{1}} \times \cdots \times G_{k_{n}}$, the multiplication taking place in $G_{k_{p}}$. And second, if $\left(g_{1}, \ldots, g_{n}\right)$ is such that $g_{p}$ lies

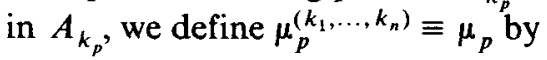

$$
\mu_{p}\left(g_{1}, \ldots, g_{n}\right)=\left(g_{1}, \ldots,\left(i_{k_{p}, k_{p+1}}\left(g_{p}\right) g_{p+1}\right), \ldots, g_{n}\right)
$$

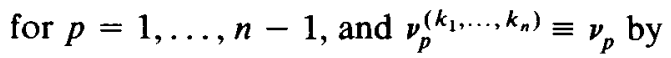

$$
\nu_{p}\left(g_{1}, \ldots, g_{n}\right)=\left(g_{1}, \ldots,\left(g_{p-1} i_{k_{p}, k_{p-1}}\left(g_{p}\right)\right), \ldots, g_{n}\right) \text { for } p=2, \ldots, n \text {. }
$$

By means of these three classes of functions we can describe all possible reductions of a non-reduced $n$-tuple in $W_{n}$ to a (reduced or non-reduced) $(n-1)$-tuple. Further, it is clear that, for each $p$ (and each $k_{1}, \ldots, k_{n}$ ), each $\lambda_{p}$, $\mu_{p}$ and $\nu_{p}$ has closed domain and is continuous.

Now we see easily from the definition of $W_{n}$ that $W_{n} \times W_{n}=\sqcup G_{i, m}^{i, j}$, where the disjoint union is over all $i, j \leqslant n$ and (for each fixed $i$ and $j$ ) all positive integers $l_{1}, \ldots, l_{i}, m_{1}, \ldots, m_{j}$, and where $G_{l, m}^{i, j}$ is shorthand for $\left(G_{l_{1}} \times \cdots \times G_{l_{l}}\right)$ $\times\left(G_{m_{1}} \times \cdots \times G_{m_{j}}\right)$ (with $l$ standing for $\left(l_{1}, \ldots, l_{i}\right)$ and $m$ for $\left.\left(m_{1}, \ldots, m_{j}\right)\right)$. To show that $\Gamma_{n}=\Gamma \cap\left(W_{n} \times W_{n}\right)$ is closed in $W_{n} \times W_{n}$, it therefore suffices to show that $\Gamma_{i, m}^{i, j}=\Gamma \cap G_{l, m}^{i, j}$ is closed in $G_{l, m}^{i, j}$ for all $i, j, l, m$. We need to distinguish four cases: (a) $i, j<n$; (b) $i=n, j<n$; (c) $i<n, j=n$; and (d) $i=j=n$.

In case (a), $G_{i, m}^{i, j}$ in fact lies in $W_{n-1} \times W_{n-1}$, so that $\Gamma_{i, m}^{i, j}=\Gamma \cap G_{i, m}^{i, j}=\Gamma_{n-1}$ $\cap G_{l, m}^{i, j}$, which is closed in $G_{i, m}^{i, j}$ by the inductive assumption.

In case (b), we claim that $\Gamma_{l, m}^{i, j}=\cup_{\sigma}(\sigma \times \iota)^{-1}\left(\Gamma_{n-1}\right)$, where $\iota$ is the identity on $G_{m_{1}} \times \cdots \times G_{m_{j}}$, and where $\sigma$ runs through all the functions of $\left\{\mu_{p}: p=\right.$ $1, \ldots, n-1\}$, of $\left\{\nu_{p}: p=2, \ldots, n\right\}$, and of $\left\{\lambda_{p}: p\right.$ satisfies $\left.l_{p}=l_{p+1}\right\}$ (with the superscripts $\left(l_{1}, \ldots, l_{n}\right)$ assumed). For if $\left(w, w^{\prime}\right) \in \Gamma_{l, m}^{i, j}$ (with $\left.i=n, j<n\right)$, then $w$ must be non-reduced, since $p(w)$ and $p\left(w^{\prime}\right)$ have the same length; and then one of the functions $\sigma$ just listed, when applied to $w$, gives $w^{\prime \prime} \in W_{n-1}$ satisfying $p(w)=p\left(w^{\prime \prime}\right)$, so that $(\sigma \times \imath)\left(w, w^{\prime}\right)=\left(w^{\prime \prime}, w^{\prime}\right) \in \Gamma_{n-1}$. Thus $\Gamma_{l, m}^{i, j}$ $\subset \cup(\sigma \times \imath)^{-1}\left(\Gamma_{n-1}\right)$. Conversely, if $\left(\sigma(w), w^{\prime}\right) \in \Gamma_{n-1}$ for some $\left(w, w^{\prime}\right) \in G_{l, m}^{i, j}$, then we must have $p(w)=p(\sigma(w))$, so that $\left(w, w^{\prime}\right) \in \Gamma_{l, m}^{i, j}$. Hence $\Gamma_{l, m}^{i, j}=$ $\bigcup_{o}(\sigma \times \imath)^{-1}\left(\Gamma_{n-1}\right)$, as claimed. Since all the functions $\sigma \times \imath$ are continuous on closed subsets of $G_{l, m}^{i, j}$, and since $\Gamma_{n-1}$ is closed in $W_{n-1} \times W_{n-1}$ by assumption, it follows that $\Gamma_{l, m}^{i, j}$ is a finite union of closed sets, and is therefore closed in $G_{l, m}^{i, j}$. Case (c) is obviously dealt with similarly.

Finally, consider case (d). If $\left(w, w^{\prime}\right) \in \Gamma_{l, m}^{i, j}$, then since the lengths of $p(w)$ and $p\left(w^{\prime}\right)$ are equal, $w$ and $w^{\prime}$ are either both reduced or both non-reduced. If, for any $p(1 \leqslant p \leqslant n)$, we have $l_{p} \neq m_{p}$, then it is clear from the lemma that $\Gamma_{i, m}^{i, j}$ can 
contain no pairs $\left(w, w^{\prime}\right)$ in which $w$ and $w^{\prime}$ are reduced. An argument like that for case (b) then shows that $\Gamma_{i, m}^{i, j}=\bigcup_{\sigma, \tau}(\sigma \times \tau)^{-1}\left(\Gamma_{n-1}\right)$, where $\sigma$ runs through the functions specified in case (b), and $\tau$ runs through a set of functions specified analogously, with (assumed) superscripts $\left(m_{1}, \ldots, m_{n}\right)$. It follows (with the assumption $l_{p} \neq m_{p}$ for some $p$ ) that $\Gamma_{l, m}^{i, j}$ is closed. Now suppose that $l_{p}=m_{p}$ for $p=1, \ldots, n$. We claim that under this assumption $\Gamma_{l, m}^{i, j}=K\left(l_{1}, \ldots, l_{n}\right) \cup$ $\bigcup_{\sigma, \tau}(\sigma \times \tau)^{-1}\left(\Gamma_{n-1}\right)$, with $\sigma$ and $\tau$ as above. To prove this, consider $\left(w, w^{\prime}\right) \in \Gamma_{l, m}^{i, j}$. If $w$ and $w^{\prime}$ are reduced, then the lemma shows that $\left(w, w^{\prime}\right) \in K\left(l_{1}, \ldots, l_{n}\right)$, while if $w$ and $w^{\prime}$ are not reduced, then $\left(w, w^{\prime}\right) \in(\sigma \times \tau)^{-1}\left(\Gamma_{n-1}\right)$ for suitable $\sigma$ and $\tau$, as earlier. Conversely, if $\left(w, w^{\prime}\right) \in K\left(l_{1}, \ldots, l_{n}\right)$, then the last part of the lemma shows that $\left(w, w^{\prime}\right) \in \Gamma_{i, m}^{i, j}$, while $\left(w, w^{\prime}\right) \in(\sigma \times \tau)^{-1}\left(\Gamma_{n-1}\right)$ implies that $\left(w, w^{\prime}\right) \in$ $\Gamma_{i, m}^{i, j}$, much as in case (b). Therefore $\Gamma_{l, m}^{i, j}$ is again a finite union of closed sets, and hence is closed.

Thus $\Gamma_{i, m}^{i, j}$ is closed in $G_{l, m}^{i, j}$ for all $i, j, l, m$, whence $\Gamma_{n}$ is closed in $W_{n} \times W_{n}$. The proposition now follows by induction.

From Proposition 4.25 of [7] (or Proposition A.1 of [1]), we may immediately deduce the following result, using the fact that $W$ is a $\mathrm{k}_{\omega}$-space.

COROllary. With the quotient topology determined by $p, G$ is a (Hausdorff) $k_{\omega}$-space.

Continuity of the group operations in $G$ now follows by a standard argument (cf. [1], [7]) which uses the facts that $p: W \rightarrow G$ and $p \times p: W \times W \rightarrow G \times G$ are both quotient maps of $\mathbf{k}_{\omega}$-spaces ([1], [7]). It also follows routinely that $G$ has the universal property required of it by the definition. It thus remains only to show that the restriction of $p$ to $G_{j}$ is a closed embedding, for each $j$. This is achieved by a simple inductive argument, modelled on that given above, which shows that if $C$ is a closed subset of $G_{j}$ for any $j$, then $p^{-1}(p(C))$ is closed in $W$, so that $p(C)$ is closed in $G$. The outline of this argument is as follows. Write $\Delta=p^{-1}(p(C))$. Now $\Delta \cap W_{1}=\sqcup_{m}\left(\Delta \cap G_{m}\right)$, and clearly $\Delta \cap G_{m}$ is $C$ if $m=j$, and is $i_{j, m}\left(C \cap A_{j}\right)$ otherwise. Therefore $\Delta \cap W_{1}$ is closed. We now assume that $\Delta \cap W_{n-1}$ is closed for some $n \geqslant 2$ and show that $\Delta \cap W_{n}$ is closed. To do this, it suffices to show that $\Delta \cap\left(G_{k_{1}} \times \cdots \times G_{k_{n}}\right)$ is closed for every choice of $k_{1}, \ldots, k_{n} \in \mathbf{N}$. But it is easy to see that $\Delta \cap\left(G_{k_{1}} \times \cdots \times G_{k_{n}}\right)=$ $\cup_{\sigma} \sigma^{-1}\left(\Delta \cap W_{n-1}\right)$, with the functions $\sigma$ as defined earlier, and so the result follows.

This completes the proof of the theorem.

As mentioned in the introduction, we can now provide a new proof of the following result of $\mathrm{La}$ Martin [6]; our proof is a topologized version of the original proof ([5]; see also [9]) that epimorphisms of groups are surjective. 
COROLlARY. Epimorphisms in the category of (Hausdorff) $\mathrm{k}_{\omega}$-groups have dense image.

Proof. Let $f: H \rightarrow G$ be an epimorphism of $\mathrm{k}_{\omega}$-groups, and let $A$ be the closure of $f(H)$ in $G$; thus $A$ is a $\mathrm{k}_{\omega}$-group. Now let $\phi_{1}, \phi_{2}$ be the two natural topological isomorphisms from $G$ into the topological amalgamated free product $G *{ }_{A} G$. Clearly $\phi_{1}$ and $\phi_{2}$ agree on, and only on, $A$. Hence $\phi_{1} f=\phi_{2} f$, and so, since $f$ is an epimorphism, $\phi_{1}=\phi_{2}$. This implies that $A=G$, that is, that $f(H)$ is dense in $G$.

\section{References}

[1] R. Brown and J. P. L. Hardy, "Subgroups of free topological groups and free topological products of topological groups", J. London Math. Soc. (2) 10 (1975), 431-440.

[2] Elyahu Katz and Sidney A. Morris, "Free products of topological groups with amalgamation", to appear.

[3] Elyahu Katz and Sidney A. Morris, "Free products of topological groups with amalgamation. II", to appear.

[4] Eli Katz and Sidney A. Morris, "Free products of $k_{\omega}$-topological groups with normal amalgamation", Topology Appl. 15 (1983), 189-196.

[5] A. G. Kurosh, A. Kh. Kivshits and E. G. Schul'geifer. "The foundations of the theory of categories", Russian Math. Surveys 15 (No. 6) (1960), 1-46.

[6] W. F. La Martin, "On the foundations of $k$-group theory", Dissertationes Math. (Rozprawy Mat.) 146 (1977).

[7] B. L. Madison, Congruences in topological semigroups (Second Florida Symposium on Automata and Semigroups, University of Florida (1971), Part II).

[8] B. H. Neumann, "An essay on free products of groups with amalgamations", Philos. Trans. Royal Soc. London (A) 246 (1954), 503-554.

[9] Eric C. Nummela, "On epimorphisms of topological groups", General Topology and Appl. 9 (1978), 155-167.

[10] Edward T. Ordman, "Free $k$-groups and free topological groups", General Topology and Appl. 5 (1975), 205-219.

[11] Barbara V. Smith Thomas, Categories of topological groups (Presented to Second Symposium on Categorical Topology, University of Capetown (1976), Memphis State University Report 76-16).

\section{Department of Mathematics \\ University of Queensland}

St. Lucia 4067

Australia 